Gerlinde Huber-Rebenich, Christian Rohr, Michael Stolz (Hrsg.)

Wasser in der

mittelalterlichen Kultur /

Water in Medieval

Culture

Gebrauch - Wahrnehmung - Symbolik /

Uses, Perceptions, and Symbolism 


\section{Das Mittelalter Perspektiven mediävistischer Forschung}

Beihefte

Herausgegeben von

Ingrid Baumgärtner, Stephan Conermann und Thomas Honegger

\section{Band 4}


Die Drucklegung dieser Publikation wurde gefördert durch:

Burgergemeinde Bern

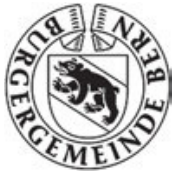

Fondation Johanna Dürmüller-Bol

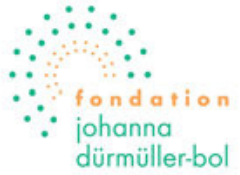

Mediävistenverband e.V.
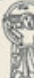

Mediävistenverband e.V.

Schweizerische Akademie der Geistes- und Sozialwissenschaften (SAGW)

Schweizerische Akademie der Geistes- und Sozialwissenschaften Académie suisse des sciences humaines et sociales Accademia svizzera di scienze umane e sociali Academia svizra da scienzas moralas e socialas Swiss Academy of Humanities and Social Sciences

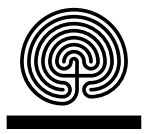

Schweizerischer Nationalfonds (SNF)

\section{FN $\mathcal{N F}$}

SCHWEIZERISCHER NATIONALFONDS

ZUR FÖRDERUNG DER WISSENSCHAFTLICHEN FORSCHUNG

\section{UniBern Forschungsstiftung \\ (Berne University Research Foundation)}

ISBN 978-3-11-044286-1

e-ISBN (PDF) 978-3-11-043743-0

e-ISBN (EPUB) 978-3-11-043476-7

\section{Library of Congress Cataloging-in-Publication Data}

A CIP catalog record for this book has been applied for at the Library of Congress.

Bibliografische Information der Deutschen Nationalbibliothek

Die Deutsche Nationalbibliothek verzeichnet diese Publikation in der Deutschen Nationalbibliografie; detaillierte bibliografische Daten sind im Internet über http://dnb.dnb.de abrufbar.

(C) 2017 Walter de Gruyter GmbH, Berlin/Boston

Satz: Dörlemann Satz GmbH \& Co. KG, Lemförde

Druck und Bindung: Hubert \& Co. GmbH \& Co. KG, Göttingen

@ Gedruckt auf säurefreiem Papier

Printed in Germany

www.degruyter.com 


\title{
Providenz und Souveränität. Wasser als Element göttlichen und menschlichen Wirkens im ,Gregorius“ Hartmanns von Aue
}

\begin{abstract}
Throughout Hartmann von Aue’s ‘Gregorius’, there are numerous instances of the narrator or characters interpreting story events as the results of divine or diabolic intervention. Nevertheless, there are also passages which stress the individual's power to act and the necessity of taking one's own decisions within - and in addition to - the context of super-natural influence. Both divine providence and human agency are represented through the image of the element of water. Thus, water functions as a sphere where divine providence and human sovereignty overlap and it illustrates a decisive characteristic of Gregorius' path to redemption, which is neither the result of unmitigated predetermination nor of pure autonomy, but the product of their interaction. The key to Gregorius' salvation lies in his own initiatives and his reactions to contingent experiences, which require independent actions and self-reliant choices.
\end{abstract}

Keywords: Hartmann von Aue, Gregorius, Providenz, Souveränität, Transzendenz

In der altgermanistischen Forschung wurde bereits verschiedentlich auf die Bedeutung des Elements Wasser für den ,Gregorius“ Hartmanns von Aue hingewiesen. Zum einen wurde wiederholt der christliche Symbolgehalt von Wasser und damit verbundener Figuren und Objekte hervorgehoben. ${ }^{1}$ Da die einzelnen Stationen auf dem Lebensweg des Protagonisten stets mit einer Passage über ein Gewässer verknüpft sind, verwundert es nicht, dass das Hauptaugenmerk zum anderen auf der durch das Wasser gegliederten Struktur, der „maritime[n] Topographie“2 der Erzählung liegt. So erkennen etwa Edith WENZEL und Horst WENZEL bezüglich des ,Gregorius“ im Wasser „das ungeordnete, bewegliche Element, das den Wechsel von einem Lebenszustand $\mathrm{zu}$ einem anderen signalisiert“. ${ }^{3}$ Peter STROHSCHNEIDER spezifiziert

1 Walter OHцу, Die heilsgeschichtliche Struktur der Epen Hartmanns von Aue, Berlin 1958, S. 37; Hinrich SiEfKEn, Der sælden strâze. Zum Motiv der Zwei Wege bei Hartmann von Aue, in: Euphorion 61 (1967), S. 1-21, hier S. 12; Ulrich ERNST, Der ,Gregorius‘ Hartmanns von Aue. Theologische Grundlagen - legendarische Strukturen - Überlieferung im geistlichen Schrifttum (Ordo 7), Köln, Weimar, Wien 2002, S. 92, 101-104.

2 Albrecht Hausmann, Gott als Funktion erzählter Kontingenz. Zum Phänomen der ,Wiederholung ‘ in Hartmanns von Aue Gregorius, in: Cornelia HeRberIcHS u. Susanne ReICHLIN (Hgg.), Kein Zufall. Konzeptionen von Kontingenz in der mittelalterlichen Literatur (Historische Semantik 13), Göttingen 2010, S. 79-109, hier S. 106.

3 Edith Wenzel u. Horst WenZel, Die Tafel des Gregorius. Memoria im Spannungsfeld von Mündlichkeit und Schriftlichkeit, in: Harald HAFERLAND u. Michael MECKLENBURG (Hgg.), Erzählungen in Er- 
diesen Befund zu der Feststellung, im ,Gregorius“ fungiere das Wasser als ein „Zwischen- und Schwellenbereich“, der die höfische Welt und die geistlich konnotierte Welt trenne und aus dem sich für die Erzählung „strukturell ein Diesseits und ein Jenseits des Wassers“4 entfalte. Die Hauptfigur überschreitet diese Schwelle im Laufe der Handlung viermal: bei der Aussetzung des inzestuös gezeugten Kindes auf dem Meer, bei der Abkehr vom Kloster als Ort der Jugend und dem Wiedereintritt in das höfische Leben, beim Rückzug auf die einsame Felseninsel als Buße für den unwissentlich begangenen Inzest mit der eigenen Mutter und schließlich bei der Rückkehr in die Welt nach der Berufung zum Papst.

Wasser stellt im ,Gregorius“ einen Übergangsbereich zwischen höfischer und geistlicher Weltorientierung dar, und es dient auch als Element, in dem sowohl göttliche als auch menschliche Handlungsmacht sichtbar und wirksam werden. Besonders augenfällig ist die Funktion des Wassers als Medium transzendenter Einflussnahme auf den Lebensweg des Helden. Von der Zeugung der Hauptfigur unter den Einflüsterungen Satans bis zu den Wundererscheinungen auf Gregorius' Weg nach Rom als designierter Papst ziehen sich Kommentierungen des Erzählers oder der Figuren durch den gesamten Text, die das Handlungsgeschehen als Ergebnis von Interventionen göttlicher oder teuflischer Macht interpretieren - und fast immer gehen diese überirdischen Lenkungen durch das Medium Wasser vonstatten. Erscheint das Leben des Protagonisten aufgrund der Vielzahl solcher Einflussnahmen als das Ergebnis kontingenter Vorgänge bzw. göttlicher Providenz ${ }^{5}$, so zeigen sich gleichzeitig aber auch die Möglichkeiten, die menschlicher Handlungskompetenz im Rahmen dieser externen Einwirkungen zukommt. Der Beziehung zwischen lenkendem Gott und handelndem Menschen als Grundthema im ,Gregorius“ dient Wasser als Leitmotiv, dessen Symbolgehalt das zentrale Thema spiegelt: Ebenso wie Wasser im ,Gregorius‘ nicht allein als ein Element dargestellt wird, dessen Gewalt der Mensch hilflos ausgeliefert wäre, erscheint auch die transzendente Lenkung, als deren Medium das Wasser fun-

zählungen. Phänomene der Narration in Mittelalter und Früher Neuzeit (Forschungen zur Geschichte der älteren deutschen Literatur 19), München 1996, S. 99-114, hier S. 111.

4 Peter StrohschneIder, Inzest-Heiligkeit. Krise und Aufhebung der Unterschiede in Hartmanns ,Gregorius', in: Christoph Huber, Burghart WACHINGER u. Hans-Joachim ZIEgELER (Hgg.), Geistliches in weltlicher und Weltliches in geistlicher Literatur des Mittelalters, Tübingen 2000, S. 105-133, hier S. 109-111.

5 Providenz und Kontingenz sind in Bezug auf mittelalterliche Literatur im Allgemeinen und Hartmanns ,Gregorius‘ im Speziellen als enge Verwandte anzusehen, insofern Gott als für alle Ereignisse verantwortliches Agens stets präsent erscheint; vgl. dazu auch Armin ScHulz, Erzähltheorie in mediävistischer Perspektive, hrsg. v. Manuel BRAUn, Alexandra DUNKEL u. Jan-Dirk MÜLlER, Berlin, Boston 2012, S. 298 (Hervorhebung im Original): „Providenz und Kontingenz bilden im Mittelalter kein gleichwertiges Gegensatzpaar, sondern eine Hierarchie [...]. Kontingenz ist nur im Kleinen möglich, unterhalb des göttlichen Heilsplans; in der Summe alles Einzelnen jedoch manifestiert sich die göttliche Providenz, auch wenn dies die menschliche Erkenntniskraft übersteigen kann.“ 
giert, nicht absolut, sondern es tun sich stets auch Freiräume auf, die der Protagonist nach eigener Entscheidung ausfüllen muss.

Gleich zu Beginn der Haupthandlung, bei der Aussetzung des Kindes Gregorius auf dem Meer, verweist der Erzähler auf eine biblische Referenzfigur, in der sich die Verbindung zentraler Themen des ,Gregorius‘ mit dem Motiv des Wassers spiegelt:

\author{
unser herre got der guote \\ underwant sich sîn ze huote, \\ von des genâden Jônas \\ ouch in dem mere genas, \\ der drîe tage und drîe naht \\ in dem wâge was bedaht \\ in eines visches wamme. (v. 929-935) ${ }^{6}$
}

Die Verknüpfung der Episode mit der Jona-Geschichte irritiert zunächst, erinnert das in seiner Kiste auf den Wellen ausgesetzte Kind doch viel eher an die Figur des Mose als an Jona. In der Forschung stehen bezüglich der Jona-Referenz zumeist die typologische Vorausdeutung von Gregorius' Auserwähltenstatus und die Parallelität der wunderbaren Errettung im Fokus. ${ }^{7}$ Doch ist damit noch nicht hinreichend die auffällige Wahl Jonas als Vergleichspunkt erklärt, da beide Aspekte auch mit Hilfe der im thematischen Kontext passenderen Mose-Figur hätten illustriert werden können. Der Mehrwert des Jona-Analogons indes liegt darin, dass hier zwei Themenkomplexe verhandelt werden, die für den ,Gregorius` zentral sind, wenn auch mit je unterschiedlicher Akzentuierung: In beiden Texten geht es zum einen um die Frage der Reichweite bzw. Universalität von Gottes Gnade, zum anderen um die Auseinandersetzung des Menschen mit göttlicher Providenz und um die Möglichkeiten individueller Initiative. ${ }^{8}$

Der zweite Aspekt erfährt seine Insbildsetzung über das Element Wasser, das hierfür aufgrund seiner stofflichen Eigenschaften wie seines bildlichen Potentials den idealen Symbolträger darstellt. Im ,Gregorius‘ bildet die Verknüpfung von Wassersymbolik und Providenzdiskurs ein den Text durchziehendes Leitmotiv, beginnend bereits mit der Aussetzung des noch ungetauften Kindes, dessen Existenz aufgrund der Umstände seiner Zeugung vor der Welt verheimlicht werden muss. Die Entschei-

6 Zit. nach Hartmann von Aue, Gregorius, hrsg. v. Hermann PAUL, neu bearb. v. Burghart WACHINGER (ATB 2), 15. Aufl. Tübingen 2004.

7 Vgl. z.B. ERNST (Anm. 1), S. 115; Volker MERTENS, Kommentar, in: Hartmann von Aue, Gregorius, Der arme Heinrich, Iwein, hrsg. u. übers. v. DEMs. (Bibliothek des Mittelalters 6), Frankfurt / M. 2004, S. 769-1108, hier S. 851; Waltraud FriTsCH-RöSSLER, Stellenkommentar, in: Hartmann von Aue, Gregorius. Mittelhochdeutsch / Neuhochdeutsch, hrsg., übers. u. komm. v. DERS. (RUB 18764), Stuttgart 2011, S. 246-307, hier S. 271.

8 Vgl. dazu Rüdiger Lux, Jona. Prophet zwischen ,Verweigerung und ,Gehorsam‘. Eine erzählanalytische Studie (Forschungen zur Religion und Literatur des Alten und Neuen Testaments 162), Göttingen 1994, S. 162; Friedemann W. GoLKA, Jona, 2. Aufl. Stuttgart 2007, S. 64. 
dung über (ewiges) Leben oder (ewigen) Tod wird dem Ratschluss Gottes überlassen, und in der Tat wird die Errettung des Kindes aus der sturmumtosten See vom Erzähler als Eingreifen Gottes dargestellt, wenn es heißt, daz die wilden winde das Kind wurfen swar in got gebôt, / in daz leben ode in den tôt (v. 926-928).

Doch Gottes Einflussnahme erschöpft sich nicht mit dem Einholen der Kiste in das Boot zweier Fischer. Stattdessen scheint es, als ob nicht nur das Überleben des Kindes, sondern auch dessen Aufnahme in das Kloster der Planung Gottes folgt, wenn der Erzähler kommentiert, dass der Abt des im Boot versteckten Kindes wart innen / von unsers herren minnen (v. 1013f.). Auf diese Weise wird eine besondere Bindung des Abtes an das Findelkind begründet, die in der eigenhändigen Durchführung der Taufe und zugleich auch der Patenschaft des Abtes für Gregorius seine Fortsetzung findet.

Nachdem der Klosterschüler Gregorius durch einen Zufall erfährt, dass er der inzestuösen Beziehung zweier Geschwister entstammt, wird das Muster der Überantwortung des Schicksals an göttliche Fügung wieder aufgerufen, wenn Gregorius sich mit dem Wunsch nach Ritterschaft und der Suche nach seinen Eltern von der Klosterinsel verabschiedet. An Bord seines Schiffes bittet er darum, daz in unser herre / sande in etelîchez lant / dâ sîn vart wære bewant (v. 1828-1830), und gibt seinen Schiffsleuten den Befehl, daz si den winden wæren / nâch ir willen undertân / und daz schef liezen gân / swar ez die winde lêrten / und anders niene kêrten (v. 1832-1836). Als das Schiff daraufhin vom Wind in ein Land getragen wird, in dem sich eine Landesherrin der Bedrängnis eines Usurpators erwehren muss, erkennt Gregorius eine gottgegebene Möglichkeit ritterlicher Bewährung. An dieser Stelle ist von Seiten des Erzählers keine Rede davon, dass Gott die Route des Schiffes bestimmt habe, sondern diese Sicht ist allein Interpretation des Protagonisten selbst. Damit setzt Gregorius zum einen Gottes Hilfestellung unreflektiert voraus ${ }^{9}$ und reduziert zum anderen seine eigenen Wünsche an seine Reise auf den Aspekt ritterlichen Kampfes. Die Enthüllung seiner Herkunft ist für ihn plötzlich nicht mehr von Interesse, folglich kommt es Gregorius nicht in den Sinn, dass ihm dieser Wunsch erfüllt wurde und es sich bei der Landesherrin um seine eigene Mutter handeln könnte.

So erlangt Gregorius zum Dank für seine Hilfe im Kampf die Hand der Herrscherin, ohne dass Mutter oder Sohn von ihrer Verwandtschaft ahnen. Für den Fortgang der Handlung spielt erneut Wasser eine Rolle: Täglich zieht sich Gregorius zurück, um heimlich die Schrifttafel zu betrachten, die ihm bei seiner Aussetzung beigegeben wurde. Eines Tages beobachtet eine Dienerin sein tränenreiches Jammern und berichtet ihrer Herrin davon. Gregorius’ Klagen verweisen auf eine im Inneren der Figur wirkende Kraft, die mit ,Wasser‘ in Form von Tränen ins Bild gesetzt wird und die seine Emotionen nach außen sichtbar macht. Die Aufdeckung des neuerlichen Inzests bewirkt eine Erschütterung seiner aktuellen Lebenssituation; in der Folge

9 So auch ERNSt (Anm. 1), S. 93. 
dieses Umbruchs ergreift Gregorius selbst die Initiative, lässt das weltliche Leben hinter sich und zieht als armer Büßer in die Wildnis.

Für seine Buße wird nun Wasser zum zentralen Element: Von einem Fischer wird er auf einer einsamen Felseninsel ausgesetzt und angekettet, der Schlüssel wird mit der höhnischen Ankündigung ins Meer geworfen, sollte dieser jemals wiedergefunden werden, sei dies der Beweis für Gregorius' Sündenbefreiung und Heiligkeit. Die unwirtliche Insel bietet die Kulisse für ein Wunder, das die schützende Hand Gottes über Gregorius erkennen lässt:

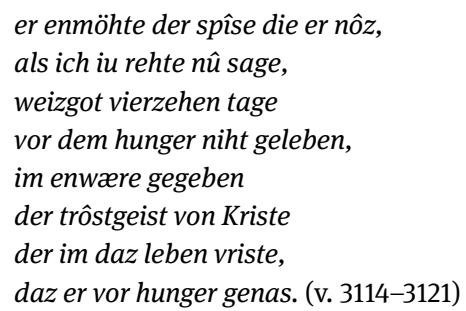

Ein aus einer Felsspalte quellendes Rinnsal, das sich jeden Tag zu einer kleinen Mulde voll Wasser sammelt, stellt Gregorius' einzige Nahrung dar, die ihn dennoch über siebzehn Jahre hinweg am Leben erhält. Mit seiner Buße auf der Felseninsel wird Gregorius zum Sinnbild des christlichen Menschen: Umgeben vom Meer der sündigen Welt, dem Sündenmeer, wird der Mensch vor dem Ertrinken gerettet durch das Vertrauen auf den Felsen Christus und damit auf die Gnade Gottes. Das irdische Wasser wird ,als sündhaft, gefährlich und todbringend“ angesehen - ein verbreitetes Motiv, an das der Erzähler anknüpft, wenn er von den vil tiefen ünden / totlîcher sünden (v. 2483 f.) spricht, in die die des Inzests schuldige Mutter versunken sei. ${ }^{10}$ Parallel zu dieser Vorstellung vom sündhaften Wasser existiert aber auch das Bild vom Wasser des Lebens, dem lebendigen Brunnen Christi, ${ }^{11}$ von dem zu trinken zugleich Ausdruck des Glaubens und Mittel der Erlösung ist. Unter Berücksichtigung dieser ambivalenten christlichen Symbolik des Wassers erscheint Gregorius' Aufenthalt auf dem Felsen aufgrund des täglichen Trinkens des Wassers des Lebens als beständiger Nachweis des Glaubens und der Buße, die ihn letztendlich aus dem Sündenmeer und dem Wasser des Todes zu erretten vermag.

Diese Errettung erfolgt wiederum durch göttliche Weisung: Nachdem man sich in Rom nicht auf die Wahl eines neuen Papstes einigen kann, entschließt man sich,

10 ERnst (Anm. 1), S. 101.

11 Heimo Reinitzer, Wasser des Todes und Wasser des Lebens. Über den geistigen Sinn des Wassers im Mittelalter, in: Hartmut BöHME (Hg.), Kulturgeschichte des Wassers (Suhrkamp-Taschenbuch 1486), Frankfurt / M. 1988, S. 99-144, hier S. 106f., 108 f.; vgl. auch Bernhard BLume, Lebendiger Quell und Flut des Todes. Ein Beitrag zu einer Literaturgeschichte des Wassers, in: DERs., Existenz und Dichtung. Essays und Aufsätze, ausgew. v. Egon SchwARZ, Frankfurt / M. 1980, S. 149-166, hier S. 152. 
auf ein Zeichen Gottes zu warten, wer neuer Bischof von Rom werden solle. Daraufhin erhalten zwei Römer im Traum die Nachricht, der neue Papst sei auf einem einsamen Felsen in Aquitanien zu finden. Die beiden werden auf die Suche geschickt und erreichen schließlich das Haus des Fischers, der Gregorius vor Jahren auf dem Felsen aussetzte. In Erwartung eines lohnenden Geschäfts bewirtet der Fischer die Gesandten mit einem frisch gefangenen Fisch. In dessen Bauch wird der einst ins Meer geworfene Schlüssel der Fußfesseln wiedergefunden und es bewahrheitet sich die spöttische Prophezeiung des Fischers:

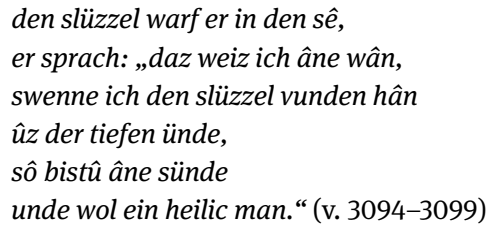

Das Medium der Verkündigung göttlichen Willens und der Mitteilung von Gregorius' Auserwähltheit und Sündenvergebung ist damit erneut das Wasser. So wie der Fisch mit der Goldmünze im Maul im Neuen Testament (Mt. 17,27) als der die Schuld der Menschheit begleichende Jesus gedeutet wird, ${ }^{12}$ so beweist der Schlüssel im Inneren des gefangenen Fischs im ,Gregorius‘ die mitgeteilte Sündenvergebung für den Protagonisten und seine Eltern; der Fischer wird damit unwissentlich zum Propheten der Heiligkeit des neuen Papstes.

Dass sich im ,Gregorius‘ göttliche Einflussnahme und Lenkung stets mit Hilfe von Wasser manifestieren, ist kein Zufall, denn, so Hans BlumenberG, „[u]nter den elementaren Realitäten, mit denen es der Mensch zu tun hat, ist ihm die des Meeres [...] die am wenigsten geheuere. Für sie sind Mächte und Götter zuständig, die sich der Sphäre bestimmbarer Gewalten am hartnäckigsten entziehen“. ${ }^{13}$ Die Gründe für die Zuschreibung des Wassers als Element des Transzendenten sind vielfältig: Zum einen ist das Wasser der Verfügungsgewalt besonders des mittelalterlichen Menschen enthoben; vor allem im Bereich der Schifffahrt war das Ausgeliefertsein an nicht kontrollierbare Kräfte deutlich spürbar. Zum anderen waren Gewässer nur schwer erkundbar, die Welt unter der Oberfläche blieb naturkundlichen Blicken verborgen; nicht umsonst wurden Fabelwesen außer an den Peripherien der bekannten Welt vor allem in den Tiefen des Meeres oder von Seen verortet. Und schließlich erscheint Wasser durch den Kreislauf aus Verdunstung und Regen ganz konkret als Stoff der Vermittlung zwischen Himmel und Erde.

12 Marianne SAmmER, Fisch, in: Metzler-Lexikon literarischer Symbole, 2. Aufl. 2012, S. 121 f. 13 Hans Blumenberg, Schiffbruch mit Zuschauer. Paradigma einer Daseinsmetapher (SuhrkampTaschenbuch Wissenschaft 289), Frankfurt / M. 1979, S. 9. 
Indem alle wichtigen Wendepunkte der Handlung als Eingriffe transzendenter Mächte durch das Medium Wasser inszeniert werden, erscheinen die Figuren im ,Gregorius` vordergründig als Spielbälle, die den Ratschlüssen höherer Instanzen ausgeliefert sind, die für den Menschen letztlich stets unergründlich bleiben. Diese Weltsicht findet ihren Ausdruck wiederum im Bild des Lebens als Schiffsreise, auf der der Mensch dem Auf und Ab der Wellen ausgeliefert ist und allein auf göttliche Gnade hoffen kann. In der christlichen Meerfahrtsymbolik führt allein das Vertrauen darauf, dass Christus das Schiff als Steuermann in den sicheren Hafen führt, zum ewigen Heil. ${ }^{14}$ Im ,Gregorius ‘ - so scheint es - ist der Mensch also nicht Herr seines eigenen Lebensschiffs, er ist das Objekt der Gnade höherer Mächte.

Eine Lektüre, die Gregorius allein als das Objekt der Handlung, als Gegenstand transzendenten Wirkens erkennt, greift meines Erachtens jedoch zu kurz. Die Frage nach den Möglichkeiten subjektiver Handlungsfreiheit des Protagonisten im ,Gregorius' ist in der Forschung bisher eher am Rande gestellt worden. Als einer von wenigen hat sich Ulrich ERNST zu diesem Thema geäußert, und auch er kommt zu dem Ergebnis, dass Gregorius nicht „nur passiv als Instrument göttlicher Ökonomie fungiert, vielmehr ist er stets als sittlich verantwortlich handelndes Individuum in der narratio präsent, so daß man allenfalls von einem Synergismus zwischen menschlichem Tugendstreben und göttlichem Gnadenhandeln sprechen kann“. ${ }^{15}$ Auch Ingrid KASTEN betont, dass bei Hartmann im Vergleich zu seiner Vorlage das „Moment der Eigenverantwortung im ,Gregorius“ doch einen signifikanten Raum“ erlange, auch wenn gleichzeitig „der Objektstatus der Figuren nicht aufgehoben wird“; ${ }^{16}$ KASTEN relativiert diese Ansicht jedoch an anderer Stelle, wenn sie sagt, dass sich für Gregorius die „Vorstellung der Verfügbarkeit über das eigene Leben [...] als eine Illusion“17 erweise, wodurch er sich am Schluss der Erzählung allein auf die Gnade Gottes verwiesen sehe.

Die aktive Rolle, die dem Protagonisten für die Handlungsführung zukommt, kann ob der Augenfälligkeit der transzendenten Einflussnahmen leicht übersehen werden, aber der Text bietet durchaus auch Passagen, die die Handlungsspielräume und die Notwendigkeit von Entscheidungen des Einzelnen neben und innerhalb des bestehenden Rahmens göttlicher Lenkung aufzeigen, wie auch Volker MERTENS bemerkt:

14 Vgl. Sibylle Selbmann, Mythos Wasser. Symbolik und Kulturgeschichte, Karlsruhe 1995, S. 92.

15 ERNST (Anm. 1), S. 198.

16 Ingrid KASTEN, Schwester, Geliebte, Mutter, Herrscherin: Die weibliche Hauptfigur in Hartmanns ,Gregorius', in: PBB 115 (1993), S. 400-420, hier S. 404.

17 Ingrid Kasten, Subjektivität im höfischen Roman, in: Reto Luzius Fetz, Roland Hagenbüchle u. Peter Schulz (Hgg.), Geschichte und Vorgeschichte der modernen Subjektivität, Bd. 1 (European Cultures. Studies in Literature and the Arts 11.1), Berlin, New York 1998, S. 394-413, hier S. 397. 
Es geht Hartmann um die Autonomiespielräume, die Gregorius beansprucht und ausfüllt. [...] Die leidvolle Erfahrung, in Krisensituationen zwar nach allgemeinen Vorgaben, letztlich aber doch autonom handeln zu müssen, machen alle Helden Hartmanns, die Artusritter Erec und Iwein ebenso wie Heinrich und Gregorius. ${ }^{18}$

Diese Handlungsmacht ist freilich nicht als Ausdruck völliger Autonomie eines Subjekts gegenüber äußeren Einflussfaktoren zu verstehen, denn die Lenkungsmöglichkeiten des Transzendenten werden im Text an keiner Stelle - weder vom Erzähler noch von den Figuren - in Frage gestellt.

Stattdessen können die Handlungsräume des Menschen treffender mit dem Begriff der Souveränität beschrieben werden, wie ihn Gernot BöHME als Gegenentwurf zum Autonomiekonzept definiert:

Dem Ideal der Autonomie setzte ich das der Souveränität entgegen. Versteht der autonome Mensch sich von seiner Selbstbestimmung her und betrachtet alles, was an ihm diese Selbstbestimmung bedroht und infrage stellt, als ihm nicht zugehörig, so ist der souveräne Mensch derjenige, der sich etwas widerfahren lassen kann. ${ }^{19}$

,Souveränität' bezeichnet für BöHME nicht die Herrschaft des Menschen über sich oder andere, sondern vielmehr die Anerkennung der Grenzen der eigenen Selbstbestimmung und des Eingebundenseins in größere, dem Zugriff des Einzelnen enthobene Kontexte:

Der souveräne Mensch unterscheidet sich von dem autonomen gerade nicht durch eine Steigerung der Herrschaft über sich selbst noch über andere. Souveränität in diesem Sinne heißt eher, nicht über alles herrschen zu müssen. [...] Der souveräne Mensch weiß, daß er nicht die ganze Wahrheit vertritt und daß seine Form des Daseins nur einen Teil des Menschseins ausmacht. [...] Er wird mit sich selbst leben als Teil eines größeren Zusammenhanges. ${ }^{20}$

In diesem Sinne erweist sich Gregorius' Handeln als souveräne Initiative und Reaktion auf die Erfahrungen von Kontingenz und externer Lenkung, indem er gegen die transzendente Einflussnahme weder ankämpft noch sich in diese schlicht ergibt. Stattdessen nimmt Gregorius die erfahrenen Kontingenzen seiner Biographie als Herausforderung zur eigenständigen Wahl des richtigen Lebenswegs an.

Ebenso wie die Einflussnahme des Transzendenten werden auch diese Handlungsspielräume des Einzelnen mit Hilfe von Wasser ins Bild gesetzt. Das auf den ersten Blick eindeutig auf äußere Lenkung des Protagonisten zielende Leitmotiv des

18 Mertens (Anm. 7), S. 794.

19 Gernot BöHME, Ich-Selbst. Über die Formation des Subjekts, München 2012, S. 7.

20 Gernot BöHME, Anthropologie in pragmatischer Hinsicht. Darmstädter Vorlesungen (Edition Suhrkamp, N. F. 301), Frankfurt / M. 1985, S. 287 f. Freilich bedarf die Adaption von BöHMEs Konzept der Souveränität auch Anpassungen an die Gegebenheiten mittelalterlicher Literatur, so etwa hinsichtlich der Religion, vgl. ebd., S. $288 \mathrm{f}$. 
Wassers erweist sich also bei näherer Betrachtung als durchaus ambivalent. So fungiert Wasser im ,Gregorius‘ als Überlappungszone des Zusammenwirkens von göttlicher Providenz und menschlicher Handlungsmacht. Gregorius demonstriert auch die Möglichkeit, sich über durch Wasser symbolisierte Bindungen und Wegweisungen hinwegzusetzen, was etwa an Gregorius' Verhältnis zum Abt deutlich wird: Dieser wird zwar durch die Taufe zu Gregorius' geistlichem Vater, doch seinem Wunsch nach einer monastischen Karriere verweigert sich Gregorius. Die mit der Wassertaufe hergestellte rituelle Verbindung zwischen Pate und Täufling ordnet Gregorius dem eigenen Streben nach Verlassen des Klosters und Eintritt in die höfische Welt unter. Das Besteigen des Schiffs mit dem Vorsatz, ritterliche Aventiure zu suchen und die eigene Herkunft aufzudecken, entspringt Gregorius' Motivation und ist nicht (oder nur indirekt) das Ergebnis äußerer Lenkung. Die Versuche externer Einflussnahme durch den Abt, der Gregorius von seinem Vorhaben abzubringen und ihn unter Hinweis auf die Gefahr weiterer sündhafter Verfehlungen zum Verbleib im Kloster zu bewegen sucht, wehrt Gregorius vehement ab. Wenn sich Gregorius dennoch der Lenkung durch Gott unterwirft, indem er den Seeleuten den Befehl gibt, Wind und Wellen bei der Steuerung des Schiffs freien Lauf zu lassen, so darf nicht übersehen werden, dass es sich hierbei um eine freiwillige Selbstauslieferung an göttliche Fügung und damit um einen souveränen Akt der Willensäußerung handelt.

Ähnlich wie bei seinem Weggang aus dem Kloster verhält es sich mit Gregorius' Entschluss zur Aufgabe allen weltlichen Besitzes und zum Aufbruch in die Einöde nach der Entdeckung des Inzests mit der Mutter. Auch hier geht der Impuls zur Beendigung des aktuellen und zum Übertritt in einen neuen Seinszustand vom Protagonisten selbst aus. Während kurz nach der Enthüllung über Gregorius berichtet wird, sînen zorn huop er hin ze gote (v. 2608), weist er nur wenige Verse später seine Mutter und Ehefrau an, mit Hilfe umfangreicher Stiftungen die Gnade Gottes zu erwirken, und erklärt:

\author{
„[...] sus senftet sînen zornmuot \\ den wir sô gar erbelget hân. \\ ich wil im ouch ze buoze stân: [...] \\ dem lande und dem guote \\ und werltlichem muote \\ dem sî hiute widerseit.“ \\ hin tet er diu rîchen kleit \\ und schiet sich von dem lande \\ mit dürftigen gewande. (v. 2734-2750)
}

Es ist zwischen beiden Textstellen also ein Sinneswandel Gregorius' erkennbar, indem er die Verantwortung für die eingetretene Katastrophe nicht mehr bei Gott sucht, sondern in eigenen Verfehlungen erkennt. Nach einem kurzen Moment des Affekts, der ihn an den Rand der Desperatio bringt, ist Gregorius zu einer souveränen Anerkennung der göttlichen Handlungsmacht fähig. Er betont in der Folge gerade die eigene Entscheidungs- und Handlungsfähigkeit und negiert damit die Vorstellung 
einer vollständigen Prädeterminiertheit des eigenen Lebens sowie einer voraussetzungslosen göttlichen Gnade, die eigene Entscheidungen letztlich ebenso überflüssig wie sinnlos machen würde.

Dem entspricht, dass Gregorius auch die Verantwortung für seine Buße in die eigenen Hände nimmt. Ohne fremde geistliche Anleitung und aus eigener Initiative wählt er die Einsiedelei als die ihm gemäße Bußform. ${ }^{21}$ Bildlichen Ausdruck findet diese Form der Selbstbestimmung wieder mit Hilfe von Wasser, wenn es heißt: er wuot diu wazzer bî dem stege (v. 2766). Vorrangig dient dieses Motiv der Veranschaulichung von Gregorius' Entschluss, alle Bequemlichkeit zu meiden und jede Möglichkeit zur Selbstkasteiung wahrzunehmen. Doch bietet das aktive und selbstbestimmte Waten durch das Wasser auch ein Gegenbild zum passiven Befahren des Wassers auf dem Schiff, das auf äußere Lenkung angewiesen ist.

Die aktive und eigenverantwortliche Rolle, die Gregorius bei der Gestaltung seines eigenen Lebens zukommt und die die Bedeutung göttlicher Vorsehung komplementiert, zeigt sich am eindrucksvollsten bei der Wahl des schmalen Pfads, der Gregorius in die Wildnis und schließlich zu einer Fischerhütte an der Küste führt. Im Text heißt es hierzu:

\author{
Nû gie ein stîc (der was smal) \\ nâhe bî einem sê ze tal. \\ den ergreif der lîplôse man \\ und gevolgete im dan \\ unz er ein hiuselîn gesach: \\ dar kêrte der arme durch gemach. (v. 2771-2776)
}

Der Weg, der Gregorius letztendlich in die absolute Isolation auf der Felseninsel führt, ergibt sich also aus den Entscheidungen des Protagonisten. Wenn sein wundersames Überleben in der lebensfeindlichen Umgebung - wie dargestellt - als rettendes Eingreifen Gottes erkennbar ist, so darf zugleich nicht übersehen werden, dass es Gregorius selber ist, der überhaupt erst die Voraussetzungen dafür schafft, dass er diese grenzenlose Bußleistung vollbringen und schließlich die Gnade Gottes erlangen kann. Gregorius erweist sich gerade nicht als reines Objekt äußerer Vorherbestimmung, vielmehr findet er sich vor Entscheidungen gestellt, die er unabhängig treffen muss. Zwar ließe sich argumentieren, dass Gregorius’ Entschlüsse zur Veränderung seiner aktuellen Lebenssituation jeweils auf gewissermaßen göttliche Einflüsterung zurückgingen, wobei der Text hierfür freilich keinen Hinweis liefert. Doch würde auch eine solche Sichtweise an der Beobachtung, dass Gregorius’ Lebensweg als Ergebnis

21 Ähnlich auch Christoph CoRmeau u. Wilhelm STÖRmer, Hartmann von Aue. Epoche - Werk Wirkung, 3. Aufl. München 2007, S. 113; Horst BRUnNER, Die poetische Insel. Inseln und Inselvorstellungen in der deutschen Literatur (Germanistische Abhandlungen 21), Stuttgart 1967, S. 52. 
des Zusammenwirkens göttlicher Lenkung und menschlicher Handlungsmacht dargestellt wird, nichts ändern.

So bietet sich in der Zusammenschau ein ambivalentes Bild: Vordergründig erscheint Gregorius' Lebensweg als das Ergebnis transzendenter Vorherbestimmung, wobei das Element Wasser als primäres Medium der Einflussnahme und Lenkung dient. Bei genauerer Betrachtung zeigt sich jedoch, dass diejenigen Situationen, in denen der Verlauf der Handlung durch höhere Mächte beeinflusst wird, stets initiiert werden von einer aktiven Entscheidung des Protagonisten: Sein Entschluss zur Beendigung der monastischen Karriere geht der Landung im Reich der Mutter voraus; die selbstauferlegte Buße und der eingeschlagene Weg in die Wildnis führen Gregorius erst auf die Felseninsel, die ihm die Möglichkeit auf göttliche Gnade eröffnet. Die Impulse zum Wechsel des bisherigen Lebenswegs gehen also in entscheidendem Maße von Gregorius selbst aus; erst nachdem ein neuer Weg eingeschlagen ist, erfolgt eine göttliche Lenkung und Fügung.

Dieser Befund lässt sich auch auf die Gesamthandlung übertragen: Der göttliche Gnadenakt, der Gregorius vom Sünder zum erwählten Papst transformiert, ist wie alle Ratschlüsse Gottes für den Menschen letztlich unergründlich und unerklärlich. Dennoch tritt er nicht voraussetzungslos und unmotiviert ein, denn erst durch seine exorbitante Buße auf der Felseninsel und durch die Aufgabe materiellen Besitzes, sozialer Bindungen, ständischer Identität und gar der eigenen Körperlichkeit kann Gregorius von Gottes Gnade erfüllt und damit erlöst werden. Gregorius’ Weg zum Heil ist also der eines unvermeidlich sündhaften, doch nichtsdestotrotz im Angesicht transzendenter Macht souveränen Menschen, insofern er die Beschränktheit seiner eigenen Handlungsfreiheit erkennt und anerkennt, sich also im Sinne BöHMEs „etwas widerfahren lassen kann“222, ohne gegenüber der von Gott als Agens verantworteten Kontingenzerfahrungen ${ }^{23}$ in Verzweiflung $\mathrm{zu}$ verfallen. Auf diese Weise gelingt es ihm, innerhalb der vorgefundenen Zustände selbst zum aktiv Handelnden zu werden. Daraus ergibt sich die Spezifik von Gregorius’ Erlösungsweg: Dieser ist weder das Produkt absoluter Vorherbestimmung noch liegt er allein in der Hand des Protagonisten, sondern er ist das Ergebnis des Zusammenwirkens von Providenz und Souveränität, von göttlicher Lenkung und menschlicher Initiative und/oder Reaktion. Der Schlüssel zur Erlösung liegt für Gregorius nicht allein im „Rechnen mit der Unberechenbarkeit Gottes“, ${ }^{24}$ sondern in der souveränen Reaktion auf diese Erfahrung, die ein eigenständiges Handeln und die eigenständige Wahl des Bußwegs erfordert.

Dieser Befund verdichtet sich in der impliziten und expliziten aquatischen Symbolik in Hartmanns ,Gregorius': Kehrt man zurück zur bereits angesprochenen christlichen Seefahrtmetapher vom Schiff des Lebens, das von den Wellen des Schicksals

22 BöHmE (Anm. 19), S. 7.

23 Vgl. Hausmann (Anm. 2), S. 79 f., 106.

24 Ebd., S. 109. 
auf- und abgetragen wird, so fügt sich hier auch die Ambivalenz der Wassersymbolik im ,Gregorius‘ als Sinnbild des Zusammenspiels von göttlicher Providenz und menschlicher Souveränität ein. Der Entschluss zum Besteigen des Schiffs muss von Gregorius selbst ausgehen, damit die lenkenden Wellen transzendenter Mächte überhaupt wirken können. Um auf dem Meer der weltlichen Sünden in den sicheren Hafen des Heils geleitet zu werden, muss der Mensch sein Lebensschiff dem Steuermann Christus anvertrauen. Dies erfordert eine Anerkennung der göttlichen Einflussmacht ebenso wie die aktive Gestaltung der sich aus dieser ergebenden Handlungsspielräume und -erfordernisse. 